\title{
Recuperação de Recursos Audiovisuais em Portais Educacionais por meio da Adição de Metadados Semânticos
}

\author{
Ismar F. Silveira, Paulo R. S. Borges \\ Programa de Pós-Graduação em Engenharia Elétrica (PPGEE) \\ Universidade Presbiteriana Mackenzie - São Paulo, SP - Brasil \\ ismar.silveira@mackenzie.br, $71412761 @ m a c k e n z i s t a . c o m . b r$
}

\begin{abstract}
One of the major challenge in searches of educational videos is that the existing techniques and tools are not being used in combined mode so that the results are satisfactory to the user. The objective of this study is to verify if the merger and metadata recovery in audiovisual content can improve the quality of results and efficiencies of searches made by users in educational portals. As procedures, in the study presented here, it was elaborated the architecture of an educational semantic portal with added metadata to the content of educational videos and built this portal. What you may notice in this portal is that, allows users to more easily with regard to the process of searching for educational content, specifically in terms of time of the search and access to specific results.
\end{abstract}

Resumo. Um dos grandes desafios nas buscas em vídeos educacionais é que as técnicas e ferramentas existentes ainda não estão sendo utilizadas de modo combinado para que os resultados sejam satisfatórios para o usuário. $O$ objetivo deste estudo é verificar se a incorporação e recuperação de metadados em conteúdos audiovisuais pode melhorar a qualidade dos resultados e eficiência das buscas feitas pelos usuários em portais educacionais. Como procedimentos, no estudo aqui apresentado, foi elaborada a arquitetura de um portal semântico educacional com adição de metadados aos conteúdos de vídeos educacionais e construído esse portal. $O$ que se pode perceber nesse portal é que, propicia aos usuários maior facilidade no que tange ao processo de busca de conteúdos educacionais, especificamente em termos de tempo da busca e acesso a resultados pontuais.

\section{Introdução}

O acesso, a recuperação e a reutilização da gama de informações veiculadas na Internet podem ser facilitados com a codificação semântica desses conteúdos, o que tem propiciado um gerenciamento inteligente, possível por meio da web semântica ou Web 3.0 (DASIOPOULOU et al, 2010). A chamada web semântica ou web inteligente ou Web 3.0 emergiu nos anos 1990, quando foi idealizada a World Wide Web (WWW) por Tim Berners-Lee, a qual tinha por objetivo estruturar os conteúdos de modo organizado através do acréscimo da semântica, entretanto não foi possível devido às tecnologias disponíveis até então (BERNERS-LEE et al, 2001). A web semântica surgiu com a evolução de recursos web e o aumento do número de usuários e foi projetada como uma web com larga interação entre o usuário e a Internet. 


\section{CBIE-LACLO 2015}

Anais dos Workshops do IV Congresso Brasileiro de Informática na Educação (CBIE 2015)

Para a adição de semântica aos conteúdos da Web tem-se utilizado tecnologias desenvolvidas para dar significado e promover a interação entre diversos sistemas (KANELLOPOULOS, 2012). Os portais web tradicionais auxiliavam a permuta de conteúdos entre grupos de pessoas e se fizeram populares. Após, surgiram os portais semânticos que usam os padrões de representação das informações da W3C subsidiados por ontologias que promovem a organização das informações nesse ambiente (JORGE, 2005). Esses portais contribuem ainda para o compartilhamento e a reutilização dos conteúdos desses portais e permitem a construção de arquiteturas mais elaboradas de navegação, bem como, consultas mais refinadas e inferências com base em informações desses conteúdos (GRUBER, 1993).

Dentre as ferramentas utilizadas nos portais semânticos, a padronização do MPEG-7 no Multimedia Content Description Interface, por exemplo, fornece uma gama de instrumentos padronizados para o delineamento dos conteúdos audiovisuais (DASIOPOULOU et al, 2010). O MPEG-7 possui partes distintas, quais sejam: o conjunto de descritores e esquemas de descrição de conteúdos visuais e de áudio; a linguagem de definição de descrição e o esquema de descrição multimídia (MATUSHIMA, 2007). Esses instrumentos padronizados, muitas vezes, estão presentes em portais educacionais e auxiliam na obtenção e recuperação de informações desses conteúdos (MCGREAL, 2004).

Estudos como Alan et al (2008); Sebastine; Thuraisingham e Prabhakaran (2010); Kanellopoulos (2012); Lachtim (2008); Silva e Gluz (2012); Patrocinio e Ishitani (2009) mostram que, de forma análoga ao modelo da programação orientada a objetos, objetos de aprendizagem com anotação semântica apropriada podem ser armazenados em repositórios de forma a serem reutilizados e referenciados no contexto educacional. Neste estudo, toma-se como pressuposto que os recursos audiovisuais são instrumentos de amplas possibilidades de utilização em diferentes áreas, dentre as quais a educação.

Considerando esses pressupostos, teve-se por hipótese que as funcionalidades semânticas podem auxiliar na recuperação de conteúdos em vídeos educacionais em tempo real, o que pode propiciar a integração desses conteúdos e uma melhoria na qualidade dos resultados e eficiência das pesquisas feitas pelos usuários de portais educacionais. Desse modo, ao adicionar semântica nos conteúdos de vídeos educacionais pode-se facilitar o acesso a informações específicas constantes nesses vídeos, conforme o interesse dos usuários: professores e alunos. Assim, questiona-se: como arquitetar um portal semântico educacional baseado em material audiovisual?

O objetivo deste estudo é verificar se a incorporação e recuperação de metadados em conteúdos audiovisuais pode melhorar a qualidade dos resultados e eficiências das buscas feitas pelos usuários em portais educacionais.

\section{Trabalhos relacionados}

Estudos relacionados a utilização de ferramentas semânticas tem trazido contribuições para o desenvolvimento da área de tecnologia da informação. A pesquisa realizada por Alan et al. (2008) consistiu na descrição de um sistema de anotação e consulta de vídeos. Tomaram por base palavras-chave de um texto, as quais admitiram a rápida recuperação de segmentos desses vídeos. As descrições foram armazenadas em um banco de dados no formato MPEG-7. Como resultados apresentaram que foi possível 


\section{CBIE-LACLO 2015}

Anais dos Workshops do IV Congresso Brasileiro de Informática na Educação (CBIE 2015)

somar interoperabilidade no sistema construído, com estrutura flexível permitindo que as extensões importassem ontologias de domínios diferenciados.

Sebastine, Thuraisingham e Prabhakaran (2009) objetivaram prover um motor de busca semântico de conteúdos audiovisuais para armazenar, indexar e recuperar vídeos da Web. Para a indexação de diversos arquivos de vídeo mapearam os vídeos de forma individual sobre o seu arquivo RDF correspondente, para ser processado, convertido e armazenado no sistema de arquivos do Google. Em seguida, indexaram os recursos dos arquivos RDF com índices individualizados por dispositivo. Para esses autores a Web Semântica fundamenta a recuperação de conteúdo multimídia e pode auxiliar em pesquisas de conteúdo.

Rolim, Osório e Avila (2010) objetivaram propor uma infraestrutura orientada semanticamente com fins de organização de material audiovisual elaborado por brasileiros. Pretenderam aprimorar as buscas realizadas por escolas, internautas e emissoras de TV comunitárias, com base em um sistema de anotação de conteúdo, utilizando conceitos de web semântica, tecnologias como MPEG-7 e OWL. Definiram o público alvo e consideraram os objetivos de uma TV comunitária experimental, firmando parcerias com essa TV que auxiliou no aprimoramento de ontologias na anotação de forma colaborativa, no compartilhamento dos conteúdos e na validação da arquitetura proposta. Afirmaram com esse estudo que, as abordagens colaborativas e a expansão da banda larga no Brasil, contribuirão para disseminar as tecnologias digitais na produção, gravação e distribuição de conteúdo na Internet.

An e Koh (2011) buscaram construir uma ferramenta para a aquisição e classificação de conteúdo multimídia que permitisse uma comunicação de forma única entre estruturas de recuperação dos dados e anotação dos componentes, persistindo-os como MPEG-7. Construíram um sistema que permite a realização de pesquisas, anotações e navegação pelos dados multimídia, através de uma interface gráfica. Afirmaram que esse sistema interativo é caracterizado pela reutilização e extensibilidade, que de forma automática reduz a descrição semântica de um grupo de conteúdos anotados.

O estudo Gluz e Vicari (2014) propõe e avalia um modelo ontológico para representar a semântica dos objetos de aprendizagem (OA), fundamentado na análise e síntese dos resultados alcançados no projeto OBAA-MILOS, com referência ao uso de ontologias para a gerência e a representação de OA. Afirmaram que a contribuição para a evolução do quadro de pesquisas foi a proposta de um modelo que determina as propriedades e os conhecimentos intrínsecos nas atividades do ciclo de vida de um objeto de aprendizagem e a interface dessa plataforma com os ambientes virtuais de aprendizagem.

Oliveira, Siqueira e Braz (2008) propuseram uma representação da estrutura de segmentação do conteúdo presente nos objetos de aprendizagem (OA) com fins de permitir o compartilhamento desses objetos e a reutilização dos segmentos, de acordo com a estruturação do conteúdo. Os autores referiram que a semântica aplicada na construção dos OA e aos agrupamentos desses OA para a formação de aulas e/ou cursos se perde, tornando difícil o compartilhamento de materiais de instrução entre diversificados desenvolvedores de conteúdo, grupos de pesquisa ou instituições. Afirmaram que a ideia de reuso, quando é aplicada sobre o conteúdo dos objetos de aprendizagem multimídia, amplia o potencial de reutilização dos mesmos. 


\section{CBIE-LACLO 2015}

Anais dos Workshops do IV Congresso Brasileiro de Informática na Educação (CBIE 2015)

A investigação desenvolvida por Silva e Gluz (2012) objetivou criar um sistema que propiciasse mecanismos de pesquisa semântica de OA com base no alinhamento de ontologias. Apresentaram como considerações que existe uma complexidade a ser encarada na otimização no procedimento no alinhamento de ontologias. Já Lachtim et al. (2009) investigaram portais semânticos que armazenam e estruturam conteúdos com base em ontologias de domínio. Propuseram o uso dessas ontologias para assessorar a fornecer dados a portais semânticos e buscaram estabelecer consultas para um mecanismo de busca na Web baseada em uma ontologia de domínio que dá subsídio a um portal, auxiliados por um dicionário de termos. Criaram uma ontologia de domínio especificamente para um portal semântico educacional e afirmam que a utilização de templates de consulta sobre a ontologia do portal é uma boa prática, vindo a facilitar as etapas de integração e mapeamento entre essas ontologias.

Silva e Costa (2012) realizaram um estudo sobre a construção de sistemas tutores inteligentes em ambientes virtuais de aprendizagem, com base em características de desenvolvimento subsidiado no reuso e em larga escala. Desenvolveram e testaram uma linha de produto de software com base na web semântica. Os resultados apresentaram-se favoráveis na experimentação efetuada em domínios distintos, mostrando-se efetivos em relação aos aspectos em larga escala, adaptatividade e evolução.

Este estudo da literatura mostra que os recursos da web semântica estão sendo utilizados na obtenção e recuperação de informações de conteúdos em multimídia, especificamente no âmbito educacional, no qual o desenvolvimento de novas tecnologias nessa direção pode auxiliar na melhoria dos processos de localização, catalogação, recuperação e reutilização de informações contidas em portais educacionais. Entretanto não apresentam validações que mostrem que as funcionalidades semânticas, em um portal semântico educacional, auxiliam na incorporação e recuperação de metadados em conteúdos de vídeos educacionais em tempo real.

\section{Metodologia}

A caracterização deste estudo tem caráter exploratório-descritivo e qualitativo. Sustentase na revisão da literatura já existente sobre o tema. A pesquisa exploratória, segundo Gil (1991, p. 45), "objetiva propiciar maior familiaridade com o problema, tornando-o mais explícito ou mesmo construir hipóteses, como resultado principal, leva ao aprimoramento de ideias ou a descoberta de intuições".

Nessa direção, o pesquisador passa a ter um maior conhecimento em relação ao problema pesquisado. Como se trata da construção de um portal semântico educacional e integração de semântica em vídeos educacionais constantes nesse portal, trata-se de uma pesquisa exploratória de razão prática ou aplicada dos conceitos da web semântica.

O desenvolvimento desta pesquisa constou de uma revisão da literatura sobre o tema, análise dos conceitos e identificação daqueles aplicáveis a arquitetura do portal, implementação do referido portal e a título de experimentação foi adicionada semântica aos conteúdos presentes no portal construído.

Para melhor entendimento, a seguir é apresentada a descrição da arquitetura e da construção do portal semântico educacional. 
CBIE-LACLO 2015

Anais dos Workshops do IV Congresso Brasileiro de Informática na Educação (CBIE 2015)

\section{Portal Semântico Educacional}

Para a construção da arquitetura do portal semântico educacional baseado por conteúdos audiovisuais tomou-se por base conceitos de Web semântica e o padrão MPEG-7. Para Dasiopoulou et al (2010) esse padrão fornece uma grande variedade de instrumentos padronizados para o delineamento dos conteúdos audiovisuais. Da literatura estudada, Rolim, Osório e Avila (2010) que utilizaram conceitos de web semântica, tecnologias como MPEG-7 e OWL com abordagem colaborativa em um sistema de TV; e An e Koh (2011) que empregaram o MPEG-7 na construção de uma ferramenta para a aquisição e classificação de conteúdo multimídia de comunicação entre as estruturas de recuperação dos dados e anotação dos componentes.

Para agregar novas funcionalidades e adicionar semântica aos conteúdos audiovisuais do portal educacional construído no âmbito desta investigação, foram empregadas outras ferramentas: o framework Spring; a Tecnologia JavaServer FacesJSF; as bibliotecas de JavaScript e Alloy; D3.js. D3 que auxilia exibir informações aos usuários usando HTML, SVG e CSS. O HTML5 e CSS3; o framework JPA com a implementação em Hibernate e o Jena; o sistema de gerenciamento de banco de dados Oracle 10g; o servidor web Apache Tomcat que hospeda o portal; e o ambiente de desenvolvimento integrado Eclipse.

Ainda em relação às ferramentas utilizadas na construção do portal semântico educacional, buscou-se nos estudos de Sebastine; Thuraisingham e Prabhakaran (2010, p.104) a compreensão da utilidade do Jena, um framework Java comumente utilizado em web semântica. Assim, juntamente com banco de dados Stardog para web semântica, de modo a realizar a gravação e a recuperação de RDF graphs, foi utilizado o Jena de código aberto escrito em Java. Essa ação permite ao portal realizar consultas do tipo SPARQL para a obtenção das informações. Esse framework e o banco de dados Stardog oferecem suporte à OWL. O desenvolvimento foi feito utilizando o processo interativo e incremental ágil SCRUM com objetivo de garantir rápida adaptação do usuário desse portal às mudanças necessárias e a sua satisfação com o serviço e material pesquisado (scrum.org).

A arquitetura desse portal semântico educacional baseado em material audiovisual, foi elaborada por vários módulos (Figura 1), com destaque na funcionalidade de edição e obtenção de conteúdos de vídeos educacionais e contém um repositório com esse tipo de vídeos.

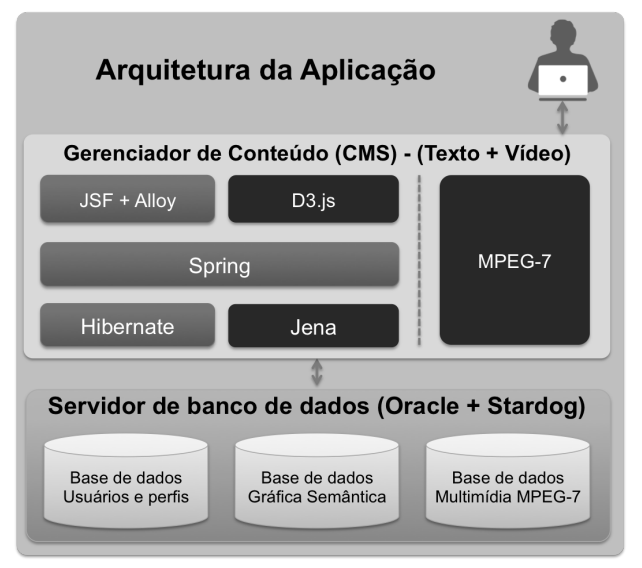

Figura 1. Arquitetura geral da aplicação 


\section{CBIE-LACLO 2015}

Anais dos Workshops do IV Congresso Brasileiro de Informática na Educação (CBIE 2015)

O armazenamento das informações está dividido em três bases: a primeira armazena as informações que auxiliam o funcionamento do portal; a segunda é a base de dados Gráfica Semântica que armazena os metadados RDFs dos conteúdos multimídia e a terceira um Multimídia Database (MMDB) que guarda o conteúdo em si dos vídeos. Para acessar os bancos, a arquitetura conta com alguns componentes de acesso a dados e todos os serviços do portal invocam esses componentes para obter informações de qualquer uma das bases.

No que tange ao gerenciamento de conteúdo, de acordo com Matushima (2007), permite a descrição de informações referentes a dados de identificação, de mídia do conteúdo e do processo de sua produção. No caso do portal semântico educacional aqui referido, os conteúdos são representados em forma de ontologia e o gerenciador de conteúdos (CMS) permitirá o cadastro de categorias e o gerenciamento do modelo de dados (estruturas) que são passíveis de utilização pelos usuários editores de conteúdo. Esse gerenciador possibilita realizar a definição das classes e suas relações. As interfaces gráficas construídas priorizam a experiência do usuário que, na maioria das vezes, é professor ou aluno. A Figura 2 apresenta a interface utilizada para essa finalidade.

Matushima (2007) refere à edição dos vídeos e suas meta-informações. Dentre outros trabalhos trazidos, o estudo de Alan et al (2008), por sua vez, refere aos usuários que almejam buscar conteúdos audiovisuais, com agilidade, esperando que a ferramenta lhes traga resultados precisos através de busca com texto livre e que encontrem um ponto certo do vídeo. O que esses usuários desconhecem é que nesse mecanismo não se considera somente a parte sintática desses conteúdos e sim, sua semântica. Lachtim et al. (2009) em seus estudos utilizaram ontologias de domínio para assessorar o fornecimento de dados a portais semânticos educacionais que permitiram a organização e a publicação dos conteúdos, de forma dinâmica no portal.

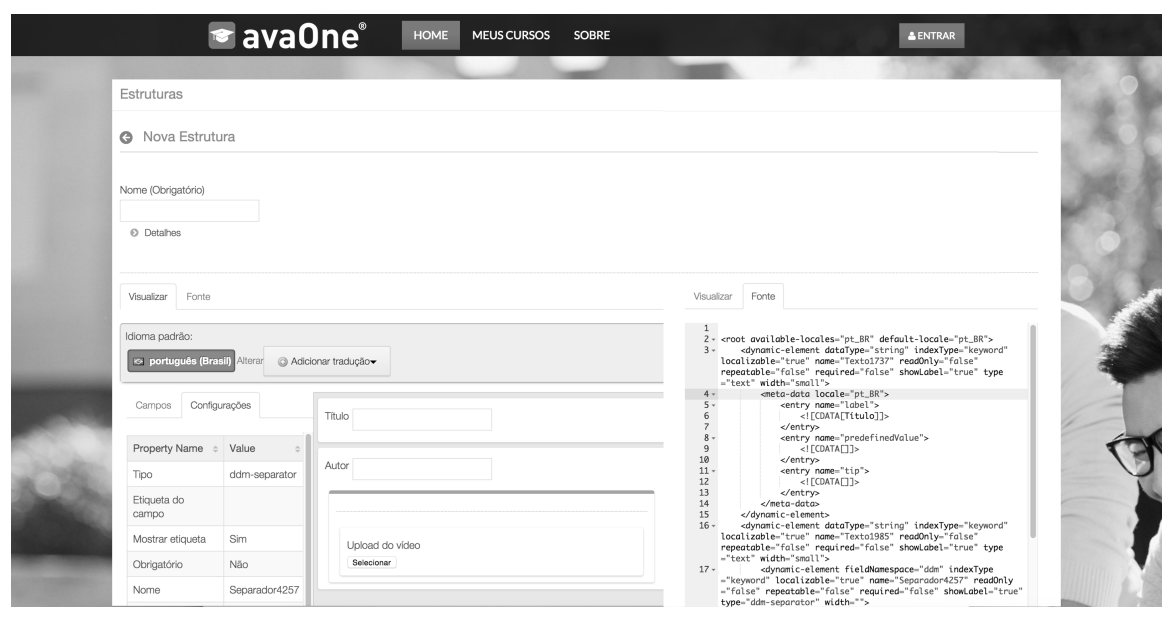

Figura 2. Interface gráfica de gerenciamento de modelos

No portal aqui apresentado foram construídos wizards para facilitar o processo de cadastro de um novo vídeo. Utilizando a especificação do MPEG-7, todas as informações produzidas como os segmentos e/ou áreas dos vídeos são armazenadas no Stardog em forma de metadados. Uma vez que as informações estão estruturadas no banco Stardog é possível realizar busca por segmentos e/ou área, por meio de critérios como: categorização, palavras-chave, segmentos ou áreas criadas por usuários do portal 


\section{CBIE-LACLO 2015}

Anais dos Workshops do IV Congresso Brasileiro de Informática na Educação (CBIE 2015)

ou alguma combinação dessas variáveis. Um usuário editor de conteúdo desse portal pode selecionar um modelo de dados e a partir dessa seleção tem a possibilidade de realização de anotações semânticas e visuais nos vídeos educacionais disponíveis. A Figura 3 apresenta a interface utilizada no processo.

As anotações visuais podem ser temporais ou de áreas e ambas permitem buscas de conteúdos e reutilização dessas informações em novos conteúdos (MARTINEZ, 2004). Além disso, ao usuário desse portal são possibilitados a navegação por categorias, o acesso e a recuperação das informações com base em uma ontologia de domínio. De acordo com Matushima (2007) o MPEG-7 fornece esquemas de descrição e descritores que auxiliam a busca de conteúdo para a navegação e o acesso dos usuários aos vídeos.

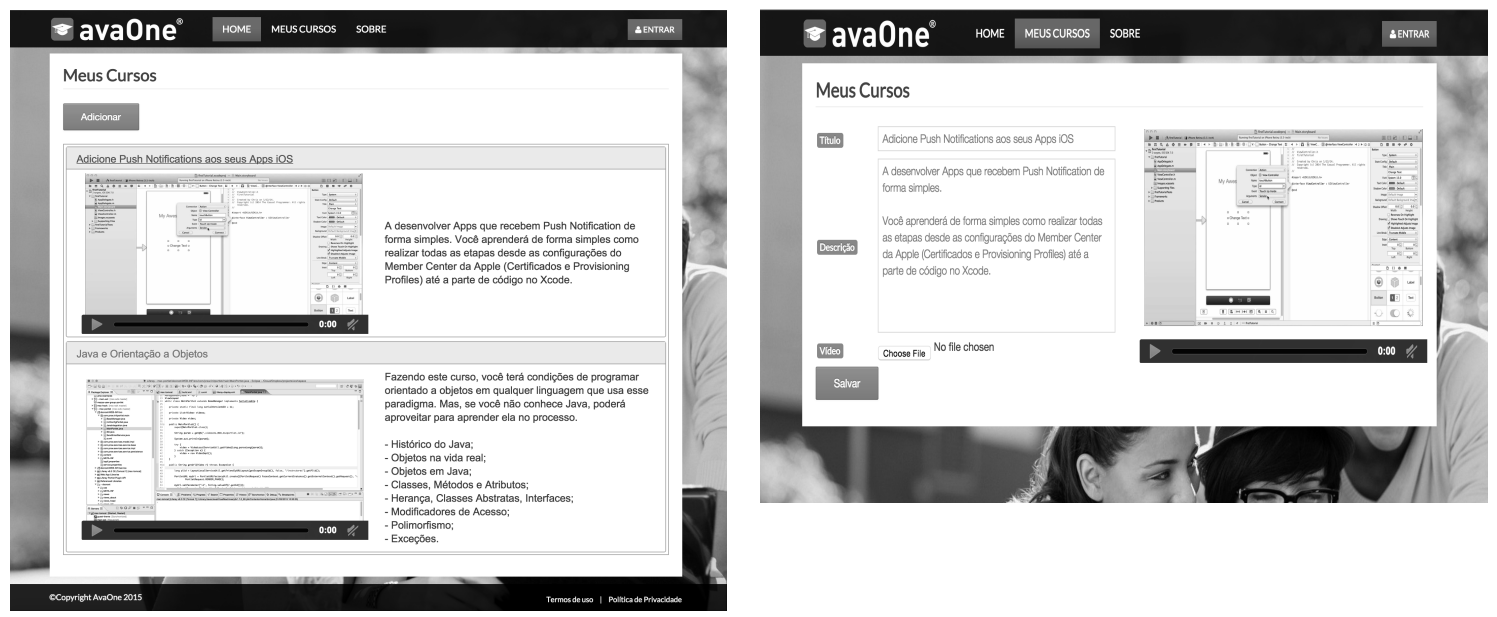

Figura 3. Interfaces utilizadas no processo de gerenciamento de vídeos

Esse portal semântico educacional possui uma página inicial que permite aos usuários usufruírem dos recursos desse portal, que disponibiliza uma área de login e de cadastro. O usuário cadastrado poderá utilizar todas as funcionalidades desse portal, relacionadas ao seu perfil. Além disso, essa página contém os vídeos educacionais mais acessados e um campo para a busca semântica.

No que se refere à busca, esse portal possui dois tipos: uma simples, na qual é exibida somente uma caixa de texto, que permite realizar uma pesquisa em todos os vídeos educacionais presentes nos bancos de dados desse portal; e a outra, avançada, que disponibiliza diversos filtros que auxiliam em uma busca mais específica, abrangendo: número de visualizações, autor, data de criação, nível de dificuldade, data de atualização e categoria.

Com base nos estudos de Alan et al (2008) para a construção do portal aqui referido, foi propiciada uma busca em modo de segmento. No entanto, a exibição dos conteúdos localizados ocorre em forma de listagem e de árvore hiperbólica, o que permite ao usuário uma navegação interativa. Na Figura 4, é exibida essa página do portal com o acesso do usuário. 


\section{CBIE-LACLO 2015}

Anais dos Workshops do IV Congresso Brasileiro de Informática na Educação (CBIE 2015)

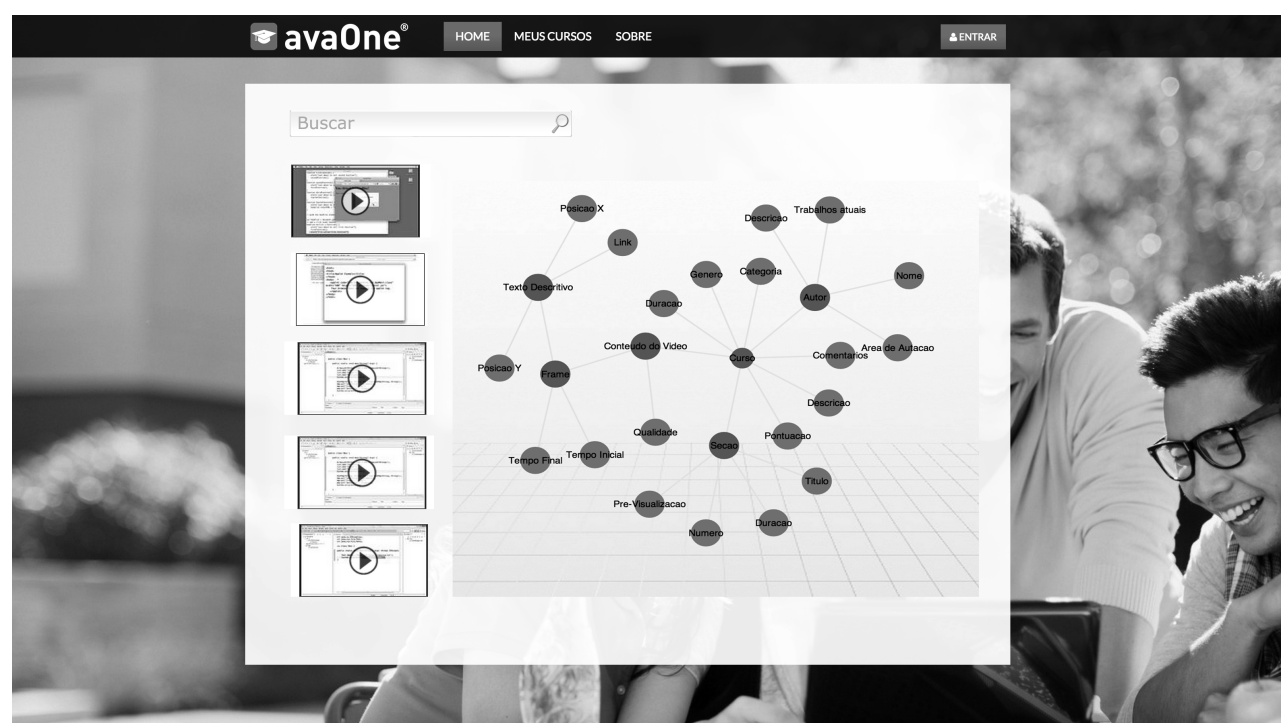

Figura 4. Resultado de buscas no portal

Em relação à interação com o usuário, vem propiciar a utilização do material multimídia (MATUSHIMA, 2007). O Portal aqui referido contém diferentes perfis para os usuários, objetivando gerenciar as funcionalidades possíveis a cada grupo de usuário. O usuário administrador tem a permissão de gerenciamento dos vídeos e meta conteúdos. Já o usuário visualizador pode somente buscar e acessar os conteúdos semanticamente. Ao clicarem no vídeo educacional escolhido, os usuários são redirecionados para a tela de detalhamento do conteúdo do vídeo, juntamente com os metadados de MPEG-7, associados a segmentos semânticos obtidos do vídeo.

Os usuários que possuem perfil para o gerenciamento de vídeos podem realizar a criação do conteúdo semântico pela interface da ferramenta de edição de conteúdo. Para tanto, são utilizados wizards para o preenchimento dos metadados associados ao padrão MPEG-7 com a finalidade de criação do conteúdo semântico, juntamente com um prévisualizador para a análise desse conteúdo. Essa construção se baseou no estudo de Alan et al (2008) no qual uma ferramenta de anotação desenvolvida para esta finalidade foi utilizada para permitir a anotação dos usuários de forma manual, sobre os conteúdos dos vídeos, o que gera os campos de dados de acordo com o contexto.

\section{Avaliação do Estudo}

O que se pode perceber é que a recuperação e incorporação de metadados nos conteúdos de portais educacionais propicia aos usuários maior facilidade no que tange ao processo de busca de conteúdos educacionais nesses portais. Especificamente em termos de tempo e pontualidade da busca, implica a melhoria na qualidade dos serviços prestados pelo portal.

Pretende-se avaliar, em caráter exploratório, a implementação do portal educacional construído por meio de testes pilotos que serão realizados na web com um grupo específico de professores e alunos do nível universitário de ensino. Aos participantes desses testes serão aplicados questionários antes e depois de sua realização. Para esses testes serão selecionados quinze vídeos relacionados a subconteúdos de linguagens de programação como: Java, PHP e C\# .net. Esses testes visam a verificação da qualidade e eficiência do sistema construído, bem como de 


\section{CBIE-LACLO 2015}

Anais dos Workshops do IV Congresso Brasileiro de Informática na Educação (CBIE 2015)

validação dos seus objetivos. Além disso para a avaliação da solução pretende-se realizar prova de conceito, seguida de validação em larga escala com o uso de repositório real.

\section{Considerações Finais}

A Realização do presente estudo até o momento, permite fazer algumas colocações. A adição de metadados em conteúdos audiovisuais e a sua recuperação são desafios na construção de portais semânticos. As técnicas e ferramentas existentes oferecem mecanismos que podem auxiliar nesse sentido, porém sua combinação, de acordo com os estudos aqui apresentados, parece ser uma limitação em sistemas para esse fim.

Trabalhos futuros podem ser realizados no sentido de estender a avaliação da eficácia desse sistema disponibilizado na web em um endereço virtual público, de maneira a validar adequadamente a eficácia do uso de mecanismos semânticos na recuperação de conteúdos audiovisuais educacionais.

\section{Referências}

ALAN, O. et al. Ontological Video Annotation and Querying System for Soccer Games. [S.1.: s.n.], 2008. 431-436 p. 23rd International Symposium on Computer and Information Sciences, Istanbul, TURKEY, OCT 27-29, 2008. ISBN 978-1-42442880-9.

AN, H.-G.; KOH, J.-J. Semantic multi-layer metadata for image annotation. [S.l.: s.n.], 2011. 1122-1124 p.

BERNERS-LEE, T.; HENDLER, J.; LASSILA, O. The Semantic Web - A new form of Web content that is meaningful to computers will unleash a revolution of new possibilities. [S.1.: s.n.], 2001. 34+ p. ISSN 0036-8733.

DASIOPOULOU, S. et al. Enquiring MPEG-7 based multimedia ontologies. [S.l.: s.n.], 2010. 331-370 p. ISSN 1380-7501.

GIL, A. C. Como elaborar projetos de pesquisa. São Paulo, v. 3, 1991.

GRUBER, T. R. Toward principles for the design of ontologies used for knowledge sharing? [S.1.]: Elsevier, 1993.907-928 p.

JORGE, M. A. Ontologias no Suporte a Portais Semânticos. Dissertação de Mestrado apresentada ao Programa de Pós-Graduação da Escola de Ciência da Informação da Universidade Federal de Minas Gerais: [s.n.], 2005. 15 p. http://www.bibliotecadigital.ufmg.br/dspace/bitstream/1843/VALA-6KFNS2/1/ mestrado__marcelo_alvim_jorge.pdf/. [Online; Acesso em: 17-dez-2011].

KANELLOPOULOS, D. Semantic annotation and retrieval of documentary media

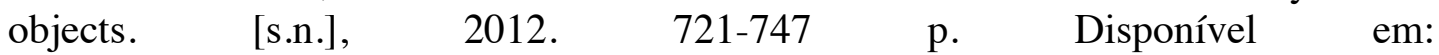
$<$ http://www.emeraldinsight.com/doi- /abs/10.1108/02640471211275756>. Acesso em: 20 fev. 2015.

LACHTIM, F. A. et al. Uma Abordagem Baseada em Ontologias para a Busca de Conteúdos na Web e Publicação em Portais Semânticos. [S.1.: s.n.], 2009.
Mpeg-7
overview (version 10).
INTERNATIONAL ORGANISATION
FOR 


\section{CBIE-LACLO 2015}

Anais dos Workshops do IV Congresso Brasileiro de Informática na Educação (CBIE 2015)

INTERNATIONALE DE NORMALISATION ISO/IEC JTC1/SC29/WG11 CODING OF MOVING PICTURES AND AUDIO, 2004. [Online; Acesso em: 20-abr-2015]. Disponível em: <http://mpeg.chiariglione.org/standards/mpeg-7>.

MATUSHIMA, R. Desenvolvimento de aplicações multimídia baseado em arquitetura orientada a serviços e nos padrões MPEG-7 e MPEG-21. 2007. Dissertação (Mestrado em Sistemas Digitais) - Escola Politécnica, Universidade de São Paulo, São Paulo, 2007. Disponível em: $<\mathrm{http}: / / \mathrm{www} . t e s e s . u s p . b r / t e s e s /$ disponiveis/3/3141/tde-08012008-150321/>. Acesso em: 2014-10-12.

MCGREAL, R., et al. (2004). EduSource: Canada's Learning Object Repository Network. The International Journal of Instructional Technology and Distance Learning, 1(3). Disponível em: <http://www.itdl.org/Journal/Mar_04/article01.htm>. Acesso: 15 mar. 2015.

OLIVEIRA, E.; SIQUEIRA, S.; BRAZ, M. H. Representação da Estruturação de Segmentos de Objetos de Aprendizagem Multimidia: Uma Abordagem Baseada em Meta-modelos de Conteúdo. [s.n.], 2008. ISSN 1414-5685. Disponível em: $<$ http://www.br-ie.org/pub/index- .php/rbie/article/view/43>.

PATROCINIO, M.; ISHITANI, L. Associacção de Recursos Semaânticos para a Anotacção de Objetos de Aprendizagem. [S.1.: s.n.], 2012.

ROLIM, L.; OSORIO, A.; AVILA, I. Collaborative System for Semantic Annotation of Audiovisual Contents - Applications in the Context of Brazilian Independent Culture. [S.1.: s.n.], 2010.1-4 p.

SEBASTINE, S. C.; THURAISINGHAM, B.; PRABHAKARAN, B. Semantic Web for Content Based Video Retrieval. [S.1.], 2009. 103-108 p. 3rd International Conference on Semantic Computing (ICSC 2009), Berkeley, CA, SEP 14-16, 2009. ISBN 978-14244-4962-0.

SILVA, Alan; COSTA, Evandro; BITTENCOURT, Ig Ibert. Uma linha de produto de software baseada na web semântica para sistemas tutores inteligentes. Revista Brasileira de Informática na Educação, v. 20, n. 1, p. 87, 2012.

SILVA, L. R. J. da; GLUZ, J. C. MSSearch: Busca Semânticção de Objetos de Aprendizagem OBAA com Suporte a Alinhamento Automático de Ontologias. [S.1.: s.n.], 2012.

VICARI, R.M; GLUZ, J. C. Rumo a uma Plataforma Semântica de Conteúdos Educacionais Digitais: o modelo ontológico. In: $25^{\circ}$ Simpósio Brasileiro de Informática na Educação (SBIE), 2014, Dourados. Anais do SBIE 2014, 2014. p. 993-1002 